\title{
Enhanced Paclitaxel Production by Addition of Water-Soluble 5-Aminolevulinic Acid and In Situ Extraction with Lauryl Alcohol in A Suspension Callus Culture
}

\author{
Kohei TAURA, Shinjiro YAMAMOTO, 'Shuhei HAYASHI and Suteaki SHIOYA \\ Department of Applied Life Science, Sojo University, 4-22-1 Ikeda, \\ Kumamoto 860-0082, Japan \\ (Received December 19, 2012; Accepted January 17, 2013)
}

\begin{abstract}
A fatty alcohol, lauryl alcohol (LA), has been found to be an effective organic solvent for in situ extraction and production of paclitaxel in a suspension plant cell culture. The culture procedure and/or conditions for enhancing paclitaxel productivity by avoiding feed-back inhibition have been studied. 5-aminolevulinic acid (ALA), which promotes plant growth, was used for this purpose in the LA-medium two phase culture systems and its effect on the paclitaxel productivity in the culture was examined. Similar callus growth and greater paclitaxel production were observed in the two phase culture systems including ALA compared to the control culture with no treatment. Enhanced paclitaxel production was recorded due to the decreased paclitaxel concentration in the medium avoiding its feed-back inhibition and the increased cellular activity by ALA. Paclitaxel productivity increased by a factor of 3 compared to the control.
\end{abstract}

\section{Introduction}

Paclitaxel $\left(\right.$ Taxol $\left.^{\circledR}\right)$, an excellent antitumor drug $[1,2]$, is produced by a semisynthetic method. However, it is very expensive because of the complex synthetic processes including a variety of reaction steps using many types of organic solvents [2]. Cell culture of Taxus species is one of the promising and greener methods because it synthesized paclitaxel through cellular metabolism without the need of much plant resources. Paclitaxel secreted into the medium from the cultured cells ceases or reduces the callus growth due to feed-back inhibition by paclitaxel. To reduce the inhibition of paclitaxel, two phase culture systems using water-immiscible organic solvents or solid absorbents such as polymeric resins [3-8] were successfully used for in situ paclitaxel recovery from the culture medium and subsequent enhancement of paclitaxel productivity. In previous research we reported that lauryl alcohol (LA) of higher $\log P$ values increased the paclitaxel productivity in the two phase systems [10-11]. Addition of an elicitor, methyl jasmonate (MJ), in the culture systems gave enhancement of paclitaxel production. However, hydrophobic MJ tends to be soluble in LA rather than the medium, which means that a large amount of MJ in the two 
phase systems is needed for this purpose [11]. For further enhancement of paclitaxel production, the use of a water-soluble chemical which promotes the plant growth and cellular activity in the two phase culture systems is of interest. The enhancement of the cellular activity might contribute to increasing production of paclitaxel. 5-aminolevulinic acid (ALA), which is a precursor in the synthesis of chlorophyll in plants, promotes growth and yield of crops and vegetables as a fertilizer [13-15]. Though ALA is supposed to have positive effects on callus suspension cultures induced from plants, there is no report on the effect of ALA on the growth and metabolite production of the cultured callus.

In the present research, the effect of ALA on the growth of callus induced from Taxus cuspidata and the secondary metabolite of paclitaxel in the LA-medium two phase culture systems was examined.

\section{Experimental}

\subsection{Callus and medium}

The callus induced from the leaves of a Taxus cuspidata tree planted in Sojo University was subcultured in a solid medium containing an agar (Gelrite, Wako Pure Chemical Co., Osaka, Japan) at $25^{\circ} \mathrm{C}$ in the dark [10]. The medium for the callus culture was a modified Gamborg's B5 [16] medium where the potassium nitrate concentration was reduced to $10 \mathrm{mM}$ and ammonium sulfate was eliminated [9].

\subsection{Lauryl alcohol and 5-aminolevulinic acid}

Lauryl alcohol (LA) was used for the in situ extraction of paclitaxel from the culture medium in the present research. LA, having a higher $\log P$ value, which means high biocompatibility and/or low cytotoxicity [17], is reported to be an effective solvent for the extraction of paclitaxel from the culture medium and to enhance paclitaxel productivity [11-13].

5-aminolevulinic acid (ALA) supplied from Cosmo oil co. was used. An ALA concentration of $1 \mathrm{mg} / \mathrm{L}$ and a light intensity of $1000 \mathrm{~lx}$, which were effective conditions selected from preliminary experiments (data not shown), were utilized for the present callus culture.

\subsection{Callus culture}

Suspension callus cultures were performed in a LA-medium two phase system as previously reported [12]. Briefly, the suspension culture, after inoculation of the precultured callus, was carried out in a 100 $\mathrm{mL}$ Erlenmeyer flask containing $20 \mathrm{~mL}$ of the medium with $10 \%$ of LA on a rotary shaker (NR-150, Taitec, Saitama, Japan) at $110 \mathrm{rpm}$ in the dark at $25^{\circ} \mathrm{C}$. The amount of fresh cells and the paclitaxel concentrations in the medium phase, the LA phase and the callus were measured in all samples.

To investigate the effectiveness of ALA and LA, the following four culture conditions were examined. All the cultures under the same conditions were performed in duplicate.

1. Callus culture including ALA alone (This culture was designated "ALA".)

2. Callus culture with in situ extraction with LA (This culture was designated "LA".)

3. Callus culture with in situ extraction with LA including ALA (This culture was designated "LA+ALA".)

4. Callus culture in the absence of LA and ALA (This culture was conducted as the control and designated "control".)

To discuss the effects of culture conditions on paclitaxel productivity, an overall production rate of paclitaxel $(O P R P)$, which is defined by the following equation, was used.

Overall production rate of paclitaxel $(O P R P)[\mu \mathrm{g}$-paclitaxel $/(\mathrm{d} \cdot \mathrm{g}$-cell $)]=\Delta \mathrm{P} /\left\{\Delta \mathrm{t} \cdot\left(F C W_{0}+F C W_{14}\right) / 2\right\}$ 
where $F C W_{0}, F C W_{14}, \triangle P$ and $\Delta t$ are the fresh cell weight at the beginning of the culture, the fresh cell weight after $14 \mathrm{~d}$ culture, the amount of produced paclitaxel during the $14 \mathrm{~d}$ culture and the culture period of $14 \mathrm{~d}$, respectively.

\subsection{Analysis}

Extraction of paclitaxel was conducted according to the procedure reported previously [12]. The extracted paclitaxel was separated by using a reversed-phase high performance liquid chromatograph system equipped with a silica-based column (Luna PFP (2), Phenomenex) and detected by UV absorbance at $225 \mathrm{~nm}$ according to the manufacture's protocol. The fresh cell weight (FCW) after harvesting, washing and drying on the filter paper was determined by weighing.

\section{Results and Discussion}

The callus growth under each culture condition including LA and/or ALA after $14 \mathrm{~d}$ culture is shown in Figure 1. Higher callus growth under all the culture conditions compared to the control was observed. Among them the growth of the callus in the culture including ALA alone was highly promoted, suggesting that ALA contributed to enhancing the growth activity of the callus. The callus growth in the LA-medium two phase culture systems was slightly smaller than that in the culture including ALA alone. The callus culture in the LA-medium two phase systems was higher than that in the control culture due to the decreased paclitaxel concentration in the medium by in situ extraction of paclitaxel with LA and subsequent decrease of feed-back inhibition of paclitaxel. In contrast, the callus growth in the LA-medium two phase systems including ALA was similar to that in the control culture, which might be due to the accumulation of excess intracellular paclitaxel as described below.

Figure 2 shows the paclitaxel concentration in the medium for each culture condition after $14 \mathrm{~d}$ culture. The paclitaxel concentration in the culture including ALA alone was higher than that in the control culture. On the other hand, the paclitaxel concentrations in the LA-medium culture systems regardless of the presence of ALA were lower than that in the control culture, resulting from in situ extraction of paclitaxel

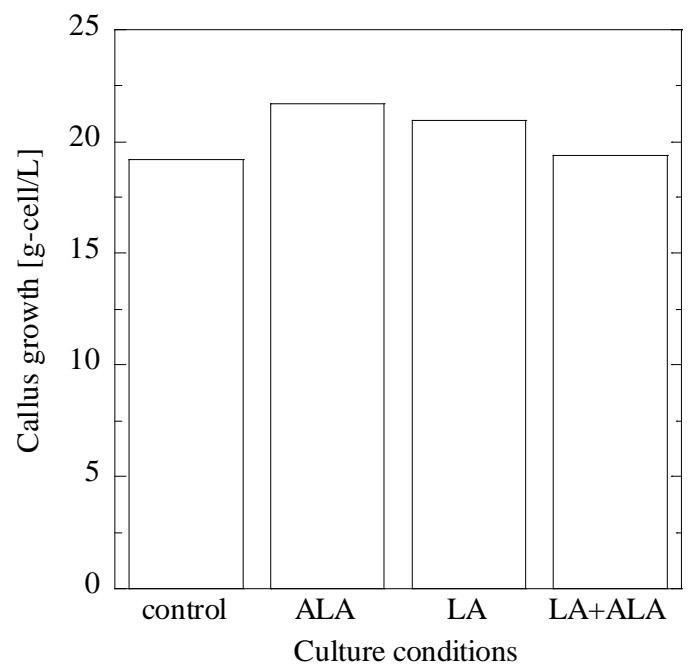

Figure 1. Comparison of callus growth under various culture conditions.



Figure 2. Paclitaxel concentration in the mediun under various culture conditions. 
in the medium with LA.

The amount of paclitaxel accumulated in the callus for each culture condition after $14 \mathrm{~d}$ culture is shown in Figure 3. The amounts of paclitaxel in the callus under all the culture conditions were greater than that for the control culture. In particular, a remarkable amount of intracellular paclitaxel in the LA-medium two phase systems including ALA was observed.

The total paclitaxel amounts for all the culture conditions were greater than that for the control culture (Figure 4). The callus cultures in the LA-medium two phase systems were effective in enhancing the total paclitaxel production compared to that in the control culture and, in particular, the culture systems including ALA enhanced paclitaxel production significantly. On the other hand, the culture including ALA alone increased paclitaxel production slightly.

ALA is known as an agent promoting growth and plant yield [13-15] and thus it is considered that ALA gave an increase of callus growth as shown in Figure 1. This result suggests that ALA acts as a potential regulator for other callus cultures for production of their valuable metabolites. Though the lowered paclitaxel concentration in the culture medium including LA resulted because of in situ extraction of paclitaxel with LA (Figure 2), little enhancement of the callus growth in the LA-medium two phase culture systems including ALA was observed, which might be due to the accumulation of excess intracellular paclitaxel (Figure 3). It is assumed that paclitaxel accumulates in the vacuole, an intracellular organelle, and thus causes insignificant inhibition on callus growth, however, the accumulation of the excess paclitaxel in the vacuole might cause an

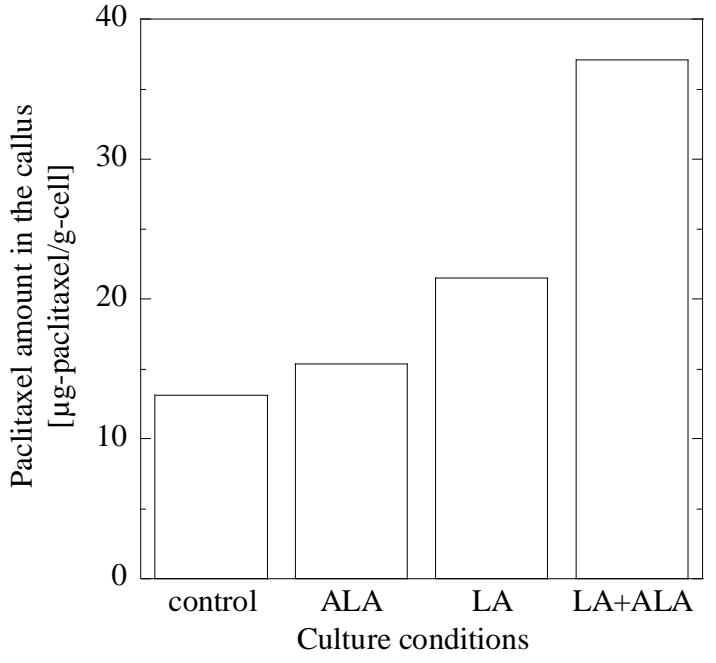

Figure 3. Comparison of paclitaxel amounts in the callus under various culture conditions.

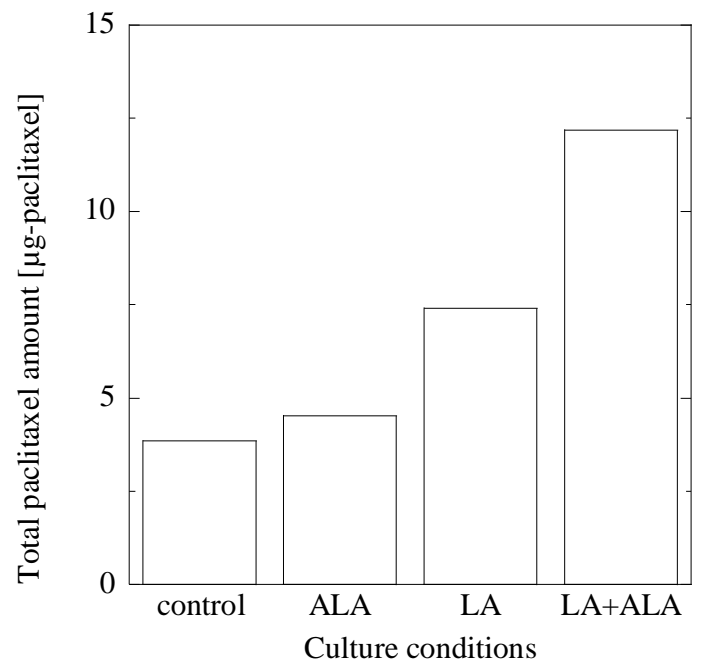

Figure 4. Comparison of total paclitaxel amounts under various culture conditions. inhibitory effect on the callus growth. This should be further examined.

To discuss paclitaxel productivity, the overall production rate of paclitaxel (OPRP) defined as Eq. (1) for each culture condition was calculated. The relationship between culture condition and $O P R P$ is shown in Figure 5. The OPRP in the culture including ALA alone was almost similar to that in the control, which might be because ALA was utilized for the callus growth rather than paclitaxel synthesis. In contrast, the $O P R P$ in the LA-medium two phase culture systems was higher. In particular, the $O P R P$ in the culture 
systems including ALA increased by a factor of 3 compared to the control culture. The reason for a higher $O P R P$ in the culture systems including LA and ALA might be that the paclitaxel concentration in the medium decreased by extraction with LA and ALA was used for paclitaxel synthesis to compensate for the decreased paclitaxel concentration in the medium rather than callus growth. This explanation is not obvious and thus should be further examined.

ALA has positive effects on callus growth and its metabolism as described above. We are now investigating the effect of light intensity on callus growth and paclitaxel production in the LA-medium two phase culture systems for an efficient culture

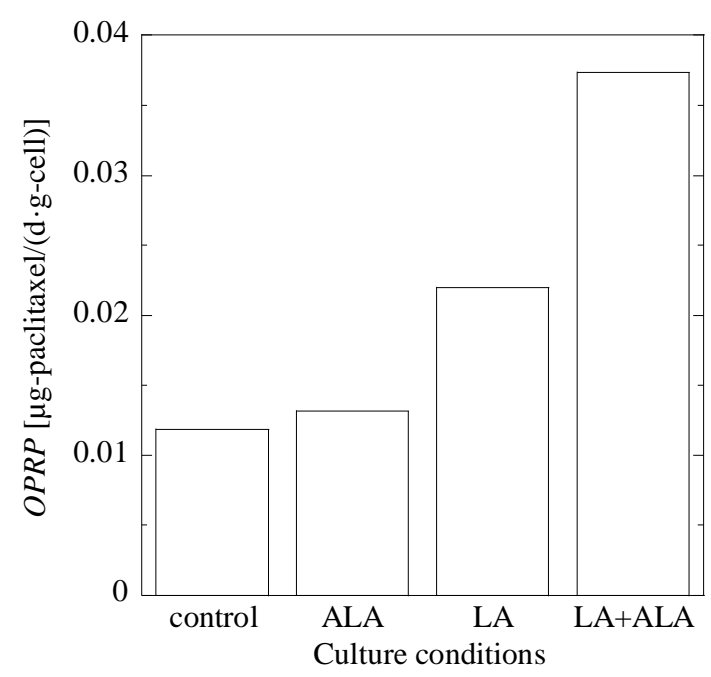

Figure 5. Comparison of the overall production rate of paclitaxel $(O P R P)$ under various culture conditions. process with higher productivity of paclitaxel. Increasing the volume fraction of LA, which improved the callus growth and paclitaxel production as previously reported [11], contributed to enhancement of the paclitaxel synthesis in the LA-medium two phase systems including ALA. Moreover, sequential refreshment of LA [12] might be also effective for this purpose in the present culture systems including ALA. Most of the paclitaxel accumulated in the callus in our experiments. Several researchers have attempted to release the intracellular paclitaxel into the medium. Brodelius reported that permeabilizing agents such as dimethyl sulfoxide and Triton X-100 tended to release the intracellular metabolites into the medium [18]. The appropriate culture conditions and/or procedure using these permeabilizing agents which secrete paclitaxel into the medium should be developed. If such a method is developed, the secreted paclitaxel would extract with LA in the two phase systems including ALA, giving effective production of paclitaxel.

\section{Conclusion}

The in situ extraction effect of lauryl alcohol (LA) including 5-aminolevulinic acid (ALA), which promotes plant growth, on callus growth and paclitaxel production in the suspension culture of $T$. cuspidata callus was investigated. The culture in the LA-medium two phase systems improved the callus growth due to the decrease of the paclitaxel concentration in the medium, i.e., decreased the feed-back inhibition of paclitaxel and enhanced paclitaxel synthesis. The culture including LA and ALA enhanced paclitaxel productivity significantly while little enhancement of callus growth was obtained due to the accumulation of the excess intracellular paclitaxel.

\section{References}

1) G.D. Kingdston, Natural taxoids: structure and chemistry. In Suffness M. ed. "Taxol: Science and Applications", Boca Raton, CRC Press, 287-315 (1995).

2) M. Suffness, Ann. Rep. Med. Chem., 28, 305-315 (1993).

3) C. Wang, J. Wu, X. Mei, Biotechnol. Prog., 17, 89-94 (2001). 
4) M. Collins-Pavao, C.-K. Chin, H. Pedersen, J. Biotechnology, 49, 95-100 (1996).

5) I.C. Kwon, Y.J. Yoo, J.H. Lee, J.O. Hyun, Process Biochemistry, 33, 701-707 (1998).

6) C. Laane, S. Boeren, K. Vos, C. Veeger, Biotechnol. Bioeng., 30, 81-87 (1987).

7) C. Wang, J. Wu, X. Mei, Appl. Microbiol. Biotechnol., 55, 404 -410 (2001).

8) T.J. McPartland, R.A. Patil, M.F. Malone, S.C. Roberts, Biotechnol. Prog., 28, 990-997 (2012).

9) M. Seki, C. Ohzora, M. Takeda and S. Furusaki, Biotechnol. Bioeng., 53, 214-219 (1997).

10) S. Yamamoto, K. Ogawa, M. Aochi, S. Hayashi, K. Nohara, S. Furusaki, Solvent Extr. Res. Dev., Jpn., 12, 149- 157 (2005).

11) S. Yamamoto, Y. Goto, T. Kawano, S. Hayashi, S. Furusaki, Solvent Extr. Res. Dev., Jpn., 13, 131-138 (2006)

12) S. Yamamoto, K. Taura, S. Hayashi, S. Furusaki, S. Shioya, Solvent Extr. Res. Dev., Jpn., 16, 93-101 (2009)

13) M.S. Naeem, H. Warusawitharana, H. Liu, D. Liu, R. Ahmad, E. A. Waraich, L. Xu, W. Zhou, Plant Physiol. Biochem., 57, 84-92 (2012).

14) R.C. Bindu, M. Vivekanandan, Plant Growth Regul., 26, 15-18 (1998) .

15) L.J. Wang, W.B. Jiang, H. Liu, W.Q. Liu, L. Kang, X.L. Hou, J. Integr. Plant Biol., 47, 1084-1091 (2005).

16) O.L. Gamborg, R.A. Miller, K. Ojima, Exp. Cell Res., 50, 151-158 (1968).

17) M. Vermue, J. Sikkema, A. Verheul, R. Bakker, J. Tramper, Biotechnol. Bioeng., 42, 747-758 (1993).

18) P. Brodelius, Appl. Microbiol. Biotechnol., 27, 561-566 (1988). 